\title{
MARKOV-MODULATED BROWNIAN MOTION WITH TWO REFLECTING BARRIERS
}

\author{
JEVGENIJS IVANOVS, ${ }^{*}$ EURANDOM, Eindhoven University of Technology \\ and University of Amsterdam
}

\begin{abstract}
We consider a Markov-modulated Brownian motion reflected to stay in a strip $[0, B]$. The stationary distribution of this process is known to have a simple form under some assumptions. We provide a short probabilistic argument leading to this result and explain its simplicity. Moreover, this argument allows for generalizations including the distribution of the reflected process at an independent, exponentially distributed epoch. Our second contribution concerns transient behavior of the model. We identify the joint law of the processes defining the model at inverse local times.
\end{abstract}

Keywords: Markov additive process; fluid model; finite buffer; two-sided reflection; inverse local time; overflow process

2010 Mathematics Subject Classification: Primary 60J55

Secondary 60K $25 ; 60 \mathrm{~K} 37$

\section{Introduction}

In this paper we investigate a Markov-modulated Brownian motion (MMBM) reflected to stay in a strip $[0, B]$, where $B>0$. Roughly speaking, an MMBM is a process with piecewise Brownian paths with drift and variance parameters determined by a finite-state Markov chain. The variance parameters are allowed to be 0 , in which case the corresponding pieces are linear. Mathematically, an MMBM is just a Markov additive process with continuous paths [4, Chapter XI]. Letting $X(t)$ be an MMBM, we construct a doubly reflected process $W(t)$ as

$$
W(t)=X(t)+L(t)-U(t),
$$

where $L(t)$ and $U(t)$ are the local times at the lower and upper barriers, respectively (that is at 0 and at $B$ ), given as the solutions of a Skorokhod problem; see Section 2.1 for details.

The model considered in this paper is also called a second-order fluid model or a fluid model with Brownian noise in the literature. It was introduced as a generalization of an extensively studied fluid flow model, where it is assumed that all the variance parameters are 0 , making the process piecewise linear. Fluid models were initially proposed for manufacturing and telecommunication systems, where units of work (products or packets) are processed so fast that they can be modeled as fluid instead of discrete units. Since then, the use of fluid models has become widespread, making it a classical model in applied probability with a variety of application areas, such as the theory of queues and dams, risk processes and insurance, environmental problems, etc. The literature on this topic is extensive, we only mention the seminal papers [2] and [10], a survey [12], and a more recent paper [1] with an extended list of references.

Received 11 May 2010; revision received 20 August 2010.

* Postal address: EURANDOM, Eindhoven University of Technology, PO Box 513, 5600 MB Eindhoven, The Netherlands. Email address: ivanovs@eurandom.tue.nl 
The second-order fluid models were simultaneously introduced in [3], [9], and [16]. The highly cited paper [16] by Rogers can be considered as one of the most influential papers, not just in the theory of fluid models, but in the much more general theory of fluctuations of Markov additive processes. In [16] the stationary distribution of a reflected MMBM is derived for both a single barrier and two barriers, assuming that all the variance parameters are either 0 or positive; see also the comments in Section 3.2. The case of a single barrier (see also [3] and [9]) is a special case of the latter problem with $B=\infty$ and is substantially easier to analyze. In fact, in this case the analysis can often be extended to more general Markov-modulated Lévy models with jumps, where all the jumps are either positive or negative; see, for example, [15], where the stationary distribution of an infinite buffer Markov-modulated M/G/1 queue is obtained.

The form of the stationary distribution of an MMBM with two reflecting barriers is strikingly simple. It is noted in [16], where the result is derived using the theory of generators of Markov processes, that there has to be a probabilistic story explaining why the solutions to the differential equations reduce so far. We provide a very simple probabilistic argument, which offers this explanation. Moreover, this argument allows us to find the stationary distribution of a reflected MMBM with both linear and Brownian segments, which to the author's knowledge has not been done so far (its transform was obtained in [7]). Furthermore, we obtain the distribution of the reflected process at an independent, exponentially distributed epoch, which gives a first insight into the transient behavior of the reflected process.

Our second contribution concerns the transient behavior of the model. We identify the joint law of the processes $t, X(t)$, and $J(t)$ at inverse local times. We envisage that this key result will find various applications (some are mentioned in Section 4). In particular, we show that a result from [7] on stationary overflow and unused capacity is a simple consequence of our result. Moreover, considering the special case of a Brownian motion we recover the results from [17].

Fluid models play a prominent role in applied probability. The importance comes from the fact that they are flexible enough to model a variety of different phenomena, and at the same time the analysis often remains tractable. One important observation is that phase-type jumps can be easily incorporated into the model. This is done by introducing auxiliary states of $J(t)$ and 'leveling' the jumps out; see, e.g. [14]. Note that this observation shows the importance of an MMBM, where some variance parameters are allowed to be 0 (the case not analyzed so far in the literature). We also remark that the level $B$ of the barrier can be interpreted in different ways. In the queueing context it is the buffer size. In the financial setting it can, for example, represent the height of a dividend barrier, in which case $U(t)$ represents the cumulative amount of dividends paid before time $t$. Throughout this paper, we use the queueing terminology.

Following some basic facts and definitions concerning two-sided reflection and MMBM given in Section 2, the paper is divided into two parts. Section 3 contains the results about the stationary distribution, and in Section 4 we discuss the inverse local times.

\section{Preliminaries}

Throughout this work, we use bold symbols to denote vectors (usually column-vectors), in particular $\mathbf{1}$ and $\mathbf{0}$ are vectors of $1 \mathrm{~s}$ and 0 s, respectively. We write $\mathbb{I}$ for the identity matrix and $\Delta_{v}$ for the diagonal matrix with entries of $\boldsymbol{v}$ on the diagonal.

\subsection{Two-sided reflection}

Let $X(t), t \geq 0$, be a real continuous function with $X(0) \in[0, B]$ (a fixed sample path of a stochastic process). The two-sided reflection $W(t)$ of $X(t)$, with respect to the strip $[0, B]$, is 
defined through (1.1), where $W(t), L(t)$, and $U(t)$ are real continuous functions which satisfy the following conditions:

- $L(t)$ and $U(t)$ are nondecreasing with $L(0)=U(0)=0$;

- $W(t) \in[0, B]$ for all $t \geq 0$;

- $W(s)=0$ if, for all $t>s, L(s)<L(t)$, and $W(s)=B$ if, for all $t>s, U(s)<U(t)$.

The last condition states that the points of increase of $L$ and $U$ are contained in $\{t \geq 0: W(t)=$ $0\}$ and $\{t \geq 0: W(t)=B\}$, respectively. It is known that such a triplet of functions exists and is unique [11], and is called the solution of the two-sided Skorokhod problem. The functions $L(t)$ and $U(t)$ are called local times at the lower and upper barriers, respectively, that is, at 0 and at $B$.

\subsection{MMBM and its basic properties}

We start with a rigorous definition of an MMBM; see also [4, Chapter XI]. Let $J(t)$ be a continuous-time irreducible Markov chain with a finite state space $E$, where $|E|=N$, and transition rate matrix $Q$. Denote its invariant distribution through $\pi$. For each $i \in E$, let $X_{i}(t)$ be a Brownian motion with variance $\sigma_{i}^{2} \geq 0$ and drift $\mu_{i}$; the vectors with elements $\sigma_{i}$ and $\mu_{i}$ are denoted through $\sigma$ and $\mu$, respectively. We assume that $J(t)$ and all the $X_{i}(t)$ are mutually independent. Letting $T$ be the last jump epoch of $J$ before $t$ ( $T=0$ if there were no jumps), we define $X(t)$ recursively as

$$
X(t)=X(T)+\sum_{i \in E} 1_{\{J(T)=i\}}\left(X_{i}(t)-X_{i}(T)\right) \quad \text { and } \quad X(0)=0,
$$

where $1_{\{\cdot\}}$ denotes the indicator function. Informally, $X(t)$ evolves as $X_{i}(t)$ while $J(t)$ is in state $i$. The law of $(X(t), J(t))$ given $J(0)=i$ is denoted through $\mathrm{P}_{i}$, and the expectation through $\mathrm{E}_{i}$. Sometimes we consider the process $\left(x_{0}+X(t), J(t)\right)$ for an arbitrary $x_{0} \in \mathbb{R}$ and still write $(X(t), J(t))$ where no confusion can arise.

Let us now introduce some important concepts related to MMBM. The asymptotic drift of the process $X(t)$ is denoted through $\kappa:=\lim _{t \rightarrow \infty} X(t) / t=\sum_{i \in E} \pi_{i} \mu_{i}$, which does not depend on the initial state of $J(t)$. Next, define the subsets $E^{+}$and $E^{-}$of $E$ through

$$
E^{ \pm}=E \backslash\left\{i \in E: \sigma_{i}^{2}=0, \pm \mu_{i} \leq 0\right\} .
$$

Thus, e.g. $E^{+}$is the set of indices of the processes $X_{i}(t)$ which are not monotonically decreasing; $X_{i}(t), i \in E^{+}$, are the processes which hit the set $(0, \infty)$ with positive probability. In addition, we let $N^{ \pm}=\left|E^{ \pm}\right|$and write $M_{ \pm}$to denote a restriction of an arbitrary matrix $M$ with $N$ rows obtained by keeping those and only those rows which are indexed by $E^{ \pm}$. Finally, $\mathbb{I}^{ \pm}$stands for the identity matrix of size $N^{ \pm}$.

Let $e_{q}$ be an exponential random variable with rate $q \geq 0$, independent of everything else, where, by convention, $e_{0}=\infty$. It is often convenient to consider the process $(X(t), J(t))$ only until $e_{q}$, at which moment it is sent to some additional absorbing state $\left(\partial_{X}, \partial_{J}\right)$-in other words, killed. The law of such a process is denoted through $\mathrm{P}^{q}$. In order to simplify notation, we drop the superscript and simply write $\mathrm{P}$ when there is no confusion. We do so in the rest of this section.

Define the first passage times for $x \geq 0$ as

$$
\tau_{x}^{ \pm}=\inf \{t \geq 0: \pm X(t)>x\}
$$


Note that $\tau_{x}^{+}=\infty$ if $X(t)$ does not exceed level $x$ before $e_{q}$. The continuity of paths and the strong Markov property of MMBM, and, in addition, the memoryless property of the exponential distribution, imply that the time-changed process $J\left(\tau_{x}^{+}\right)$is a Markov chain itself; see also [16]. Note that $J\left(\tau_{x}^{+}\right)$jumps to the absorbing state $\partial_{J}$ at $x=\sup \left\{X(t): 0 \leq t<e_{q}\right\}$. Apart from $\partial_{J}$, this Markov chain can take values only in $E^{+}$. Furthermore, it is irreducible when restricted to the set $E^{+}$. Let $\Lambda^{+}$be the corresponding $N^{+} \times N^{+}$transition rate matrix of $J\left(\tau_{x}^{+}\right)$. Also, let $\Pi^{+}$be an $N \times N^{+}$matrix with elements $\mathrm{P}_{i}\left(J\left(\tau_{0}^{+}\right)=j\right)$, where $i \in E$ and $j \in E^{+}$(with an obvious order); we often simply write $\mathrm{P}\left(J\left(\tau_{0}^{+}\right)\right)$. Using this notation, we can further write

$$
\mathrm{P}\left(J\left(\tau_{x}^{+}\right)\right)=\Pi^{+} \mathrm{e}^{\Lambda^{+} x} .
$$

The Markov chain $J\left(\tau_{x}^{+}\right)$(restricted to $E^{+}$) is recurrent if and only if $q=0$ and $\kappa \geq 0$. Finally, we consider $\tau_{x}^{-}$, and define the matrices $\Lambda^{-}$and $\Pi^{-}$in an obvious way. Note that the matrices $\Lambda^{ \pm}$and $\Pi^{ \pm}$depend on $q$. In order to stress this dependence and distinguish from the special case of $q=0$, we sometimes call them $q$-killed versions.

The matrices $\Lambda^{ \pm}$and $\Pi^{ \pm}$play a prominent role in the following. It is therefore required in practice to be able to compute these matrices. There are two methods in the literature. Firstly, we can use an iteration scheme; see, e.g. [3] and [6]. Secondly, a Jordan normal form of $\Lambda^{ \pm}$ can be obtained directly using the theory of generalized Jordan chains; see [7] and [8]. Finally, it is known that

$$
\frac{1}{2} \Delta_{\sigma}^{2} \Pi^{ \pm}\left(\Lambda^{ \pm}\right)^{2} \mp \Delta_{\mu} \Pi^{ \pm} \Lambda^{ \pm}+(Q-q \mathbb{I}) \Pi^{ \pm}=\mathbb{O},
$$

where $\mathbb{O}$ is an $N \times N^{ \pm}$matrix of 0 s; see, for example, [6].

\section{The stationary distribution}

In the first part of this section we provide an elementary derivation of the stationary distribution of $(W(t), J(t))$; we let $(W, J)$ denote the corresponding random vector. Let $\hat{J}(t)$ be the time-reversed version of $J(t)$, that is, $\hat{J}(t)$ is a Markov chain with transition rate matrix $\Delta_{\pi}^{-1} Q^{\top} \Delta_{\pi}$. Define $\hat{X}(t)$ according to $(2.1)$ with $\hat{J}(t)$ in place of $J(t)$. The law of the timereversed MMBM is denoted through $\hat{\mathrm{P}}$. We start with the following basic identity:

$$
\mathrm{P}(W \geq x \mid J=i)=\hat{\mathrm{P}}_{i}\left(X\left(\tau_{[x-B, x)}\right) \geq x\right),
$$

where $\tau[u, v)=\inf \{t \geq 0: X(t) \notin[u, v)\}$. This type of representation was first noted in [13] in the case of a random walk with two reflecting barriers. A short derivation of its continuoustime analogue is given in [4, Proposition 3.7, Chapter XIV]; see also [5] for the case of Markov additive input. It is well known that an MMBM cannot hit a level without passing it, so we obtain

$$
\hat{\mathrm{P}}(W \geq x \mid J=i)=\mathrm{P}_{i}\left(\tau_{x}^{+}<\tau_{B-x}^{-}\right), \quad \text { where } x \in(0, B] .
$$

Note that this identity does not indeed hold for $x=0$ and $i \in E \backslash E^{+}$.

Next, for $a, b \geq 0$, with at least one being strictly positive, consider the matrices

$$
C(a, b)=\mathrm{P}\left(\tau_{a}^{+}<\tau_{b}^{-}, J\left(\tau_{a}^{+}\right)\right), \quad D(a, b)=\mathrm{P}\left(\tau_{b}^{-}<\tau_{a}^{+}, J\left(\tau_{b}^{-}\right)\right),
$$

of dimensions $N \times N^{+}$and $N \times N^{-}$, respectively. The strong Markov property implies that

$$
C(a, b)=\mathrm{P}\left(J\left(\tau_{a}^{+}\right)\right)-\mathrm{P}\left(\tau_{b}^{-}<\tau_{a}^{+}, J\left(\tau_{a}^{+}\right)\right)=\mathrm{P}\left(J\left(\tau_{a}^{+}\right)\right)-D(a, b) \mathrm{P}_{-}\left(J\left(\tau_{a+b}^{+}\right)\right),
$$


where $\mathrm{P}_{-}$denotes a restriction according to $J(0) \in E^{-}$. Expand $D(a, b)$ in a similar way to arrive at

$$
\begin{aligned}
& C(a, b)=\Pi^{+} \mathrm{e}^{a \Lambda^{+}}-D(a, b) \Pi_{-}^{+} \mathrm{e}^{(a+b) \Lambda^{+}}, \\
& D(a, b)=\Pi^{-} \mathrm{e}^{b \Lambda^{-}}-C(a, b) \Pi_{+}^{-} \mathrm{e}^{(a+b) \Lambda^{-}} .
\end{aligned}
$$

Assuming that $\kappa \neq 0$ we define

$$
K^{+}:=\left(\mathbb{I}^{+}-\Pi_{+}^{-} \mathrm{e}^{B \Lambda^{-}} \Pi_{-}^{+} \mathrm{e}^{B \Lambda^{+}}\right)^{-1}, \quad K^{-}:=\left(\mathbb{I}^{-}-\Pi_{-}^{+} \mathrm{e}^{B \Lambda^{+}} \Pi_{+}^{-} \mathrm{e}^{B \Lambda^{-}}\right)^{-1},
$$

where the inverses are well defined, because one of the matrices $\Pi_{+}^{-} \mathrm{e}^{B \Lambda^{-}}$and $\Pi_{-}^{+} \mathrm{e}^{B \Lambda^{+}}$is a transient probability matrix (and the other is recurrent). Thus,

$$
C(x, B-x)=\left(\Pi^{+} \mathrm{e}^{x \Lambda^{+}}-\Pi^{-} \mathrm{e}^{(B-x) \Lambda^{-}} \Pi_{-}^{+} \mathrm{e}^{B \Lambda^{+}}\right) K^{+},
$$

and then (3.2) leads to the following result

Theorem 3.1. If $\kappa \neq 0$ then, for $x \in(0, B]$, it holds that

$$
\hat{\mathrm{P}}(W \geq x \mid J)^{\top}=\left(\Pi^{+} \mathrm{e}^{x \Lambda^{+}}-\Pi^{-} \mathrm{e}^{(B-x) \Lambda^{-}} \Pi_{-}^{+} \mathrm{e}^{B \Lambda^{+}}\right) K^{+} \mathbf{1}
$$

Finally, we give a brief comment on the more delicate case of $\kappa=0$. The problem of this case lies in the fact that the system of equations (3.3) does not identify the matrices $C(a, b)$ and $D(a, b)$ uniquely. In fact, exactly one equation is missing. This equation is of the form

$$
C(a, b)\left(a \mathbf{1}_{+}+\boldsymbol{h}_{+}\right)+D(a, b)\left(-b \mathbf{1}_{-}+\boldsymbol{h}_{-}\right)=\boldsymbol{h},
$$

where $\boldsymbol{h}$ is any vector which solves $Q \boldsymbol{h}+\boldsymbol{\mu}=\mathbf{0}$; see [7, Section 7].

\subsection{Further identities}

First, we present a very simple coupling argument, which allows us to derive an equation that complements (3.6). Consider the two-sided reflection $\tilde{W}(t)$ of $(-X(t), J(t))$ in $[0, B]$. Assuming that $X(0)=0$ and $\tilde{X}(0)=B$, it is easy to see that $\mathrm{P}(W(t) \leq x \mid J(t))=\mathrm{P}(\tilde{W}(t) \geq$ $B-x \mid J(t))$. Letting $t \rightarrow \infty$ we obtain, from (3.6),

$$
\hat{\mathrm{P}}(W \leq x \mid J)^{\top}=\hat{\mathrm{P}}(\tilde{W} \geq B-x \mid J)^{\top}=\left(\Pi^{-} \mathrm{e}^{(B-x) \Lambda^{-}}-\Pi^{+} \mathrm{e}^{x \Lambda^{+}} \Pi_{+}^{-} \mathrm{e}^{B \Lambda^{-}}\right) K^{-} \mathbf{1}
$$

where $x \in[0, B)$. Note that $\Lambda^{+}$and $\Pi^{+}$become $\Lambda^{-}$and $\Pi^{-}$, as the MMBM $(-X(t), J(t))$ is used to construct $(\tilde{W}, J)$.

Note that (3.6) and (3.7) lead to two different representations of the density $\hat{\mathrm{P}}(W \in \mathrm{d} x \mid J)$. In addition, we easily obtain the point masses at 0 and $B$ (from (3.7) and (3.6), respectively):

$$
\begin{aligned}
\hat{\mathrm{P}}(W=0 \mid J)^{\top} & =\left(\Pi^{-}-\Pi^{+} \Pi_{+}^{-}\right) \mathrm{e}^{B \Lambda^{-}} K^{-} \mathbf{1}, \\
\hat{\mathrm{P}}(W=B \mid J)^{\top} & =\left(\Pi^{+}-\Pi^{-} \Pi_{-}^{+}\right) \mathrm{e}^{B \Lambda^{+}} K^{+} \mathbf{1} .
\end{aligned}
$$

Finally, $\Pi_{+}^{+}=\mathbb{I}^{+}$and $\Pi_{-}^{-}=\mathbb{I}^{-}$, and, hence, $W$ has no mass at 0 or $B$ given $J$ is in $E^{+}$or, respectively, $E^{-}$. 


\subsection{On the result of Rogers [16]}

In this section we comment on the result of Rogers [16]. We are only interested in a fluid model with Brownian noise and a finite buffer presented in [16, Section 7]. It is assumed there that the fluid evolves as an independent sum of a Markov-modulated linear drift and a standard Brownian motion. At first sight, this is a rather special case of an MMBM. Note, however, that the process $(X(t), J(t))$ can be time changed without changing the distribution of $(W \mid J)$ in the following way. We scale time by $c_{i}>0$ while $J(t)$ is in state $i$, that is, we consider a new MMBM specified by the transition rate matrix $\Delta_{c}^{-1} Q$ and parameters $\sigma_{i}^{2} / c_{i}$ and $\mu_{i} / c_{i}$. It is easy to see that this new MMBM gives rise to the same distribution of ( $W \mid J)$. Hence, Rogers [16], in fact, does not assume more than this: all the variance parameters are strictly positive. In other words, $E^{+}=E^{-}=E$ and, hence, $\Pi^{ \pm}=\mathbb{I}$. Under this assumption, (3.6) results in the following:

$$
\mathrm{P}(W \in \mathrm{d} x \mid J)^{\top}=-\left(\mathrm{e}^{x \hat{\Lambda}^{+}} \hat{\Lambda}^{+}+\mathrm{e}^{(B-x) \hat{\Lambda}^{-}} \hat{\Lambda}^{-} \mathrm{e}^{B \hat{\Lambda}^{+}}\right)\left(\mathbb{I}-\mathrm{e}^{B \hat{\Lambda}^{-}} \mathrm{e}^{B \hat{\Lambda}^{+}}\right)^{-1} \mathbf{1},
$$

which is (7.13) of [16] up to the minus sign. Here $\hat{\Lambda}^{ \pm}=\hat{\Gamma}_{\mp}$, because of the different definition of time reversal in [16, Equation (3.3)]. The missing minus sign is a consequence of a mistake in normalization in [16]. Finally, we note that in [16] the above result was obtained using the theory of generators of Markov processes. It required solving second-order differential equations, and verification of the solution (positivity of the density is not established in [16] though). On the contrary, our result was obtained directly using elementary probabilistic arguments. This allowed us to treat the problem in its full generality, i.e. no assumption on variances.

\subsection{The distribution at an exponential epoch}

In this section we identify $\mathrm{P}_{i}\left(W\left(e_{q}\right), J\left(e_{q}\right)=j\right)$ for $X(0) \in\{0, B\}$ (start at a boundary), which provides some information of the transient behavior of the reflected process. An inspection of the proof of [4, Propostion 3.7, Chapter XIV] reveals that a representation similar to (3.1) holds true for finite time $T$ :

$$
\mathrm{P}_{i}(W(T) \geq x \mid J(T)=j)=\hat{\mathrm{P}}_{j}\left(\tau_{[x-B, x)} \leq T, X\left(\tau_{[x-B, x)}\right) \geq x \mid J(T)=i\right),
$$

where it is assumed that $X(0)=0$. Note that $\pi_{i} \mathrm{P}_{i}(J(T)=j)=\pi_{j} \hat{\mathrm{P}}_{j}(J(T)=i)$ to arrive at the equation

$$
\hat{\mathrm{P}}_{i}\left(W\left(e_{q}\right) \geq x, J\left(e_{q}\right)=j\right)=\mathrm{P}_{j}\left(\tau_{x}^{+}<e_{q}, \tau_{x}^{+}<\tau_{B-x}^{-}, J\left(e_{q}\right)=i\right) \frac{\pi_{j}}{\pi_{i}},
$$

which in matrix form can be written as

$$
\hat{\mathrm{P}}\left(W\left(e_{q}\right) \geq x, J\left(e_{q}\right)\right)^{\top}=\Delta_{\pi} \mathrm{P}^{q}\left(\tau_{x}^{+}<\tau_{B-x}^{-}, J\left(\tau_{x}^{+}\right)\right)\left[\mathrm{P}\left(J\left(e_{q}\right)\right)\right]_{+} \Delta_{\pi}^{-1} .
$$

Moreover, we can show that (3.5) holds true under exponential killing. Noting that $\mathrm{P}\left(J\left(e_{q}\right)\right)=$ $q(q \mathbb{I}-Q)^{-1}$ we find that

$$
\hat{\mathrm{P}}\left(W\left(e_{q}\right) \geq x, J\left(e_{q}\right)\right)^{\top}=q \Delta_{\pi}\left(\Pi^{+} \mathrm{e}^{x \Lambda^{+}}-\Pi^{-} \mathrm{e}^{(B-x) \Lambda^{-}} \Pi_{-}^{+} \mathrm{e}^{B \Lambda^{+}}\right) K^{+}\left[(q \mathbb{I}-Q)^{-1}\right]_{+} \Delta_{\pi}^{-1},
$$

where $x \in(0, B]$ and all the occurrences of matrices $\Lambda^{ \pm}$and $\Pi^{ \pm}$refer to the $q$-killed versions. Finally, we can derive a symmetric equation for the case $X(0)=B$. The distribution of $W\left(e_{q}\right)$ in the case of $X(0) \in(0, B)$ does not have an explicit form. In this case we need to resort to its Laplace transform, as in [7]. 


\section{Inverse local times}

This section is devoted to the study of the transient behavior of the model, which is given by $(t, X(t), J(t), W(t), L(t), U(t))$. More concretely, we characterize the joint law of these processes at inverse local times $\tau_{x}^{L}, x \geq 0$, and $\tau_{x}^{U}, x \geq 0$, where

$$
\tau_{x}^{L}=\inf \{t \geq 0: L(t)>x\} \quad \text { and } \quad \tau_{x}^{U}=\inf \{t \geq 0: U(t)>x\}
$$

for any $x \geq 0$. This key result allows us to answer a number of important questions. For example, given $X(0) \in[0, B]$ and $J(0) \in E$, when does the buffer become empty for the first time and what is the state of $J(t)$ at this time? What is the amount of lost fluid until then? Mathematically speaking, we are interested in $\left(\tau_{0}^{L}, J\left(\tau_{0}^{L}\right), U\left(\tau_{0}^{L}\right)\right)$. We can also ask: what is the length of an arbitrary busy period? What is the amount of lost fluid during a busy period given there was a loss? Moreover, we condition on the state $i$ of $J(t)$ right before this busy period starts and the state $j$ at which it finishes. These quantities are described by the jumps of $\tau_{x}^{L}$ and $U\left(\tau_{x}^{L}\right)$ given there is a corresponding transition of $J\left(\tau_{x}^{L}\right)$. The answer to these questions is immediate in view of Theorem 4.1. Moreover, in Section 4.4 we show that the stationary overflow and unused capacity vectors can be trivially obtained from Theorem 4.1; by doing so we recover a result from [7]. Finally, in Section 4.5 we consider a special case of a simple Brownian motion and recover the results from [17], where, as an easy consequence, an asymptotic variance of the overflow process is obtained.

It should be noted that the analysis of the two-sided reflection problem, compared to the one-sided problem, is considerably harder. The main problem lies in the fact that there are two local time processes, which are interrelated in an intricate way. The crucial idea is to study the set of points $x \geq 0$ such that $X\left(\tau_{x}^{L}\right)=y$ for a fixed $y \in \mathbb{R}$; see Lemma 4.1. This idea in a simpler form also appears in [16, Section 5], where it was used to derive point masses of $W(t)$ at 0 and $B$ in stationarity.

\subsection{Markov additive processes}

It will be shown that $(X(t), J(t))$ observed at inverse local times is a Markov additive process (MAP). In this section we present a definition and some basic properties of a MAP. A MAP is a bivariate Markov process $(Y(t), \tilde{J}(t))$, which satisfies the following property for every $T \geq 0$. Given $\tilde{J}(T)=i$, the shifted process $(Y(T+t)-Y(T), \tilde{J}(T+t)), t \geq 0$, is independent from $(Y(t), \tilde{J}(t)), 0 \leq t \leq T$, and has the same law as $(Y(t), \tilde{J}(t)), t \geq 0$, given $\tilde{J}(0)=i$. It is commonly assumed that the state space of $\tilde{J}(t)$ is finite; in our case it is a subset of $E$. Such a process can be seen as a Markov-modulated Lévy process with additional jumps at the transition epochs of $\tilde{J}(t)$; see [4, Chapter XI]. Hence, a MAP with continuous paths is an MMBM. Importantly, if $Y(t)$ has no negative jumps then, for all $\alpha \leq 0$, there exists a square matrix function $F(\alpha)$ such that

$$
\mathrm{E}\left[\mathrm{e}^{\alpha Y(t)} ; \tilde{J}(t)\right]=\mathrm{e}^{F(\alpha) t},
$$

where the term on the left-hand side denotes a matrix with elements $\mathrm{E}_{i}\left[\mathrm{e}^{\alpha Y(t)} 1_{\{\tilde{J}(t)=j\}}\right]$ for $i, j$ in the state space of $\tilde{J}(t)$. We can now see that $F(\alpha)$ uniquely specifies the law of the process $(Y(t), \tilde{J}(t))$. This matrix can be written explicitly in terms of the Lévy exponents of the underlying Lévy processes, the Laplace transforms of the additional jumps (at transition epochs), and the transition rate matrix of $\tilde{J}(t)$. Finally, there exists a (Perron-Frobenius) eigenvalue $k(\alpha)$ of $F(\alpha)$, which is real and larger than the real parts of all the other eigenvalues of $F(\alpha)$. 


\subsection{The main result}

We start by making the following observations:

- $\tau_{x}^{L}$ and, hence, $J\left(\tau_{x}^{L}\right), X\left(\tau_{x}^{L}\right), U\left(\tau_{x}^{L}\right)$ are also right continuous;

- $L\left(\tau_{x}^{L}\right)=x$ by the continuity of $L(t)$;

- $W\left(\tau_{x}^{L}\right)=0$, because $\tau_{x}^{L}$ is a point of increase of $L(t)$;

- $U\left(\tau_{x}^{L}\right)$ is piecewise constant;

- $X\left(\tau_{x}^{L}\right)=U\left(\tau_{x}^{L}\right)-x$ is piecewise linear.

Consider the model under the time change $t=\tau_{x}^{L}$ (and, similarly, $t=\tau_{x}^{U}$ ) and note, using the above observations, that it is enough to characterize the trivariate process $\left(\tau_{x}^{L}, X\left(\tau_{x}^{L}\right), J\left(\tau_{x}^{L}\right)\right)$. By the strong Markov property, the process $\left(\tau_{x}^{L}-\tau_{0}^{L}, X\left(\tau_{x}^{L}\right)-X\left(\tau_{0}^{L}\right), J\left(\tau_{x}^{L}\right)\right)$ is a MAP (with two-dimensional additive component) independent of $\left(\tau_{0}^{L}, X\left(\tau_{0}^{L}\right), J\left(\tau_{0}^{L}\right)\right)$. In particular, $J\left(\tau_{x}^{L}\right)$ is a Markov chain. It is an irreducible Markov chain taking values in $E^{-}$. The additive components $\tau_{x}^{L}$ and $X\left(\tau_{x}^{L}\right)$ have no negative jumps; hence, the corresponding $N^{-} \times N^{-}$matrix exponent $F^{L}(\cdot, \cdot)$ exists on $\mathbb{R}_{-}^{2}$ and the following holds:

$$
\mathrm{E}_{x_{0}}\left[\mathrm{e}^{\alpha X\left(\tau_{x}^{L}\right)-q \tau_{x}^{L}} ; J\left(\tau_{x}^{L}\right)\right]=\mathrm{E}_{x_{0}}\left[\mathrm{e}^{\alpha X\left(\tau_{0}^{L}\right)-q \tau_{0}^{L}} ; J\left(\tau_{0}^{L}\right)\right] \mathrm{e}^{F^{L}(-q, \alpha) x}
$$

for all $q \geq 0$ and $\alpha \leq 0$; see Section 4.1. Moreover, the matrix exponent $F^{L}(-q, \alpha)$ and the transform of the initial distribution $\mathrm{E}_{x_{0}}\left[\mathrm{e}^{\alpha X\left(\tau_{0}^{L}\right)-q \tau_{0}^{L}} ; J\left(\tau_{0}^{L}\right)\right]$ uniquely specifies the law of $\left(\tau_{x}^{L}, X\left(\tau_{x}^{L}\right), J\left(\tau_{x}^{L}\right)\right)$. Note that $q \geq 0$ can be seen as the rate of an independent exponential killing of the original process. In the following we do not explicitly write the killing rate $q$ in order to simplify the notation.

As shown above, the joint law of $(t, X(t), J(t), L(t), U(t))$ observed at $t=\tau_{x}^{L}, x \geq 0$, is uniquely characterized by the quantities $\mathrm{E}_{x_{0}}\left[\mathrm{e}^{\alpha X\left(\tau_{0}^{L}\right)} ; J\left(\tau_{0}^{L}\right)\right]$ and $F^{L}(\alpha)$ ( $q$ is implicit here). Hence, our goal is to determine these quantities, as well as those corresponding to $\tau_{x}^{U}$. Define $N \times N^{-}$and $N \times N^{+}$dimensional matrices $M^{L}(\alpha)$ and $M^{U}(\alpha)$ through

$$
M^{L}(\alpha)=\mathrm{E}_{x_{0}}\left[\mathrm{e}^{\alpha X\left(\tau_{0}^{L}\right)} ; J\left(\tau_{0}^{L}\right)\right]\left(F^{L}(\alpha)\right)^{-1}, \quad M^{U}(\alpha)=\mathrm{E}_{x_{0}}\left[\mathrm{e}^{\alpha X\left(\tau_{0}^{U}\right)} ; J\left(\tau_{0}^{U}\right)\right]\left(F^{U}(\alpha)\right)^{-1},
$$

for those values of $q \geq 0$ and $\alpha \leq 0$ for which the inverses are well defined. Letting $x_{0}=0$ and restricting the rows of $M^{L}(\alpha)$ to $E^{-}$, we obtain $\left(F^{L}(\alpha)\right)^{-1}$. Hence, $\mathrm{E}_{x_{0}}\left[\mathrm{e}^{\alpha X\left(\tau_{0}^{L}\right)} ; J\left(\tau_{0}^{L}\right)\right]$ and $F^{L}(\alpha)$ are readily obtained from $M^{L}(\alpha)$ (a similar statement is true with respect to $\tau_{x}^{U}$ ). We therefore aim to determine the matrices $M^{L}(\alpha)$ and $M^{U}(\alpha)$.

Let $\rho^{+}, \rho^{-}$and $k^{L}(\alpha), k^{U}(\alpha)$ be the Perron-Frobenius eigenvalues of $\Lambda^{+}, \Lambda^{-}$and $F^{L}(\alpha)$, $F^{U}(\alpha)$, respectively. It is well known that $\rho^{+}, \rho^{-} \leq 0$. If $q>0$ then the inequalities are strict. If $q=0$ and $\kappa \neq 0$, then one of the inequalities is strict depending on the sign of $\kappa$. In the following we exclude the exceptional case of $q=0$ and $\kappa=0$. We are ready to present our main result.

Theorem 4.1. Let $\alpha \in\left(\rho^{-},-\rho^{+}\right)$. Then $k^{L}(\alpha), k^{U}(\alpha)<0$ and $M^{L}(\alpha), M^{U}(\alpha)$ are uniquely specified by

$$
\begin{aligned}
& \left(M^{L}(\alpha), M^{U}(\alpha)\right)\left(\begin{array}{cc}
\mathbb{I}^{-} & -\Pi_{-}^{+} \mathrm{e}^{B \Lambda^{+}} \\
-\Pi_{+}^{-} \mathrm{e}^{B \Lambda^{-}} & \mathbb{I}^{+}
\end{array}\right) \\
& \quad=\left(\Pi^{-}\left(\Lambda^{-}-\alpha \mathbb{I}^{-}\right)^{-1} \mathrm{e}^{x_{0} \Lambda^{-}}, \Pi^{+}\left(\Lambda^{+}+\alpha \mathbb{I}^{+}\right)^{-1} \mathrm{e}^{\left(B-x_{0}\right) \Lambda^{+}}\right) .
\end{aligned}
$$


We make some comments concerning this theorem. Firstly, $k^{L}(\alpha)<0$ and $k^{U}(\alpha)<0$ ensure that the matrices $M^{L}(\alpha)$ and $M^{U}(\alpha)$ are well defined. Secondly, a simple algebraic manipulation shows that (4.2) can be equivalently rewritten as

$$
\begin{aligned}
& M^{L}(\alpha)=\left(\Pi^{-}\left(\Lambda^{-}-\alpha \mathbb{I}^{-}\right)^{-1} \mathrm{e}^{x_{0} \Lambda^{-}}+\Pi^{+}\left(\Lambda^{+}+\alpha \mathbb{I}^{+}\right)^{-1} \mathrm{e}^{\left(B-x_{0}\right) \Lambda^{+}} \Pi_{+}^{-} \mathrm{e}^{B \Lambda^{-}}\right) K^{-}, \\
& M^{U}(\alpha)=\left(\Pi^{+}\left(\Lambda^{+}+\alpha \mathbb{I}^{+}\right)^{-1} \mathrm{e}^{\left(B-x_{0}\right) \Lambda^{+}}+\Pi^{-}\left(\Lambda^{-}-\alpha \mathbb{I}^{-}\right)^{-1} \mathrm{e}^{x_{0} \Lambda^{-}} \Pi_{-}^{+} \mathrm{e}^{B \Lambda^{+}}\right) K^{+},
\end{aligned}
$$

where $K^{-}$and $K^{+}$are given in (3.4) and are well defined unless $q=\kappa=0$, which was excluded from our consideration.

Remark 4.1. Theorem 4.1 can be rewritten in a very concise way using a generalized Jordan pair $(V, \Gamma)$ of the analytic matrix function $1 / 2 \Delta_{\sigma}^{2} \alpha^{2}+\Delta_{\mu} \alpha+Q-q \mathbb{I}$; see [7]. In particular, using Lemma 6.3 of [7] we arrive at

$$
\left(M^{L}(\alpha), M^{U}(\alpha)\right)\left(\begin{array}{c}
-V_{-} \\
V_{+} \mathrm{e}^{B \Gamma}
\end{array}\right)=V(\alpha \mathbb{I}-\Gamma)^{-1} \mathrm{e}^{x_{0} \Gamma} .
$$

This expression may be used in practice when one is interested in computing the matrices $M^{L}(\alpha)$ and $M^{U}(\alpha)$.

\subsection{Proof of Theorem 4.1}

The crucial idea of the proof of Theorem 4.1 is to consider the points $x \geq 0$ such that $X\left(\tau_{x}^{L}\right)=y$ for a fixed $y \in \mathbb{R}$. Hence, we define $x^{(0)}=\inf \left\{x \geq 0: X\left(\tau_{x}^{L}\right)=y\right\}$ and $x^{(n)}=\inf \left\{x>x^{(n-1)}: X\left(\tau_{x}^{L}\right)=y\right\}$ for $n \geq 1$. Recall that $X\left(\tau_{x}^{L}\right)=\bar{U}\left(\tau_{x}^{L}\right)-x$ is piecewise linear with slope -1 , so $x^{(n)}$ is strictly larger than $x^{(n-1)}$. Equivalently, we can look at the time points $t \geq 0$, such that the local-time process $L(\cdot)$ is increasing and $X(\cdot)$ is at a fixed level $y$ at the time $t$. The following lemma provides the connection between the above mentioned points and some quantities which are easily computable.

Lemma 4.1. Let

$$
\varsigma_{y}=\inf \left\{t>\tau_{B+y}^{+}: X(t)<y\right\} .
$$

It holds almost surely that

$$
\tau_{x^{(0)}}^{L}= \begin{cases}\tau_{|y|}^{-} & \text {if } y \leq 0 \\ \varsigma_{y} & \text { if } y>0\end{cases}
$$

Moreover, for $y=0, X(0)=0$, and $J(0) \in E^{-}$, it holds almost surely that

$$
\tau_{x^{(1)}}^{L}=\varsigma_{0} .
$$

Let us provide some explanation of this result; see also Figure 1. If $y \leq 0$ then the first passage time of the level $y$ must be a point of increase of $L(t)$, otherwise $W(t)$ becomes negative. If $y>0$ then the first passage time of the level $y$ may not be a point of increase of $L(t)$. It is necessary that the buffer is empty when $X(t)$ passes level $y$, which is only possible if an overflow has occurred before. Hence, $X(t)$ should drop by at least $B$ at the time of hitting $y$, which implies that $\tau_{x^{(0)}}^{L}=\varsigma_{y}$. In order to characterize $\tau_{x^{(n)}}^{L}, n \geq 1$, we use the strong Markov property. Hence, we need to only consider the case in which $y=0$ and $X(0)=0, J(0) \in E^{-}$. In this case $\tau_{x^{(0)}}^{L}=0$ and $\tau_{x^{(1)}}^{L}=\varsigma_{0}$ by a similar argument as above. We only present a rigorous proof of this latter result. 


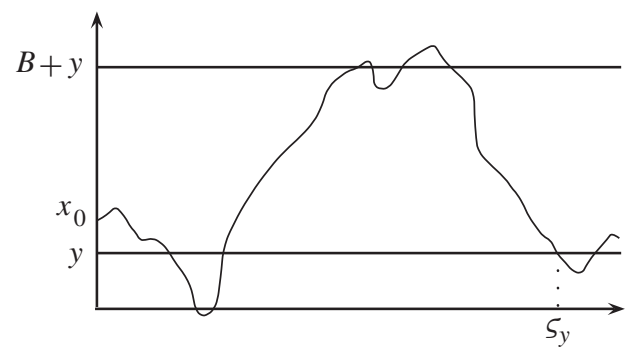

FIGURE 1: A sample path of $X(t)$.

Proof of Lemma 4.1. We assume that $y=X(0)=0$ and $J(0) \in E^{-}$, and let $\tau:=\tau_{x^{(1)}}^{L}$. First, we show that $\tau<\infty$ implies that $\varsigma_{0} \leq \tau$. Observe that $0=W(\tau)=X(\tau)+L(\tau)-U(\tau)$ and so $U(\tau)=L(\tau)>0$, because $\tau>\tau_{0}^{-}=0$. Hence, there was reflection from above before $\tau$. Let $\hat{\tau}=\sup \{t \in(0, \tau): W(t)=B\}$. Then $W(\hat{\tau})=B, U(\tau)-U(\hat{\tau})=0$, and $L(\tau)-L(\hat{\tau}) \geq 0$, because $L$ is nondecreasing. Thus, $B=W(\hat{\tau})-W(\tau)=X(\hat{\tau})+L(\hat{\tau})-L(\tau)$, and so $X(\hat{\tau}) \geq B$. But $X(t)$ cannot hit $B$ without passing it with probability 1 ; hence, $\varsigma_{0} \leq \tau$ almost surely.

Using the first part, note that if $\varsigma_{0}=\infty$ then $\tau=\infty$. Assuming that $\varsigma_{0}<\infty$ we can easily see that $\varsigma_{0}$ is a point of increase of $L(t)$. But $X\left(\varsigma_{0}\right)=0$, so by the definition of $\tau$ we have $\tau \leq \varsigma_{0}$, which concludes the proof.

Observe that

$$
\begin{gathered}
\mathrm{P}\left(J\left(\tau_{|y|}^{-}\right)\right)=\Pi^{-} \mathrm{e}^{\left(x_{0}-y\right) \Lambda^{-}} \quad \text { if } y \leq 0, \\
\mathrm{P}\left(J\left(\varsigma_{y}\right)\right)=\Pi^{+} \mathrm{e}^{\left(B+y-x_{0}\right) \Lambda^{+}} \Pi_{+}^{-} \mathrm{e}^{B \Lambda^{-}} \quad \text { if } y \geq 0,
\end{gathered}
$$

where $x_{0} \in[0, B]$. Recall the definition of $K^{-}$, which is given in (3.4), and note that it can be written as

$$
K^{-}=\sum_{n=0}^{\infty}\left(\Pi_{-}^{+} \mathrm{e}^{B \Lambda^{+}} \Pi_{+}^{-} \mathrm{e}^{B \Lambda^{-}}\right)^{n} .
$$

These facts and the strong Markov property lead to the following corollary.

Corollary 4.1. It holds that

$$
\begin{gathered}
\mathrm{E} \sum_{x \geq 0} 1_{\left\{X\left(\tau_{x}^{L}\right)=y, J\left(\tau_{x}^{L}\right)\right\}}=\Pi^{-} \mathrm{e}^{\left(x_{0}-y\right) \Lambda^{-}} K^{-}, \quad y \leq 0, \\
\mathrm{E} \sum_{x \geq 0} 1_{\left\{X\left(\tau_{x}^{L}\right)=y, J\left(\tau_{x}^{L}\right)\right\}}=\Pi^{+} \mathrm{e}^{\left(B+y-x_{0}\right) \Lambda^{+}} \Pi_{+}^{-} \mathrm{e}^{B \Lambda^{-}} K^{-}, \quad y>0 .
\end{gathered}
$$

The final step of the proof is given in the following lemma.

Lemma 4.2. For any measurable nonnegative function $f$, it holds almost surely that

$$
\int_{0}^{\infty} f\left(X\left(\tau_{x}^{L}\right)\right) 1_{\left\{J\left(\tau_{x}^{L}\right)=j\right\}} \mathrm{d} x=\int_{-\infty}^{\infty} f(y) \sum_{x \geq 0} 1_{\left\{X\left(\tau_{x}^{L}\right)=y, J\left(\tau_{x}^{L}\right)=j\right\}} \mathrm{d} y .
$$

Intuitively, this lemma states that we can interchange the 'integrals'. 
Proof of Lemma 4.2. Recall that $X\left(\tau_{x}^{L}\right)=U\left(\tau_{x}^{L}\right)-x$ and that $U\left(\tau_{x}^{L}\right)$ is piecewise constant. Suppose that $U\left(\tau_{x}^{L}\right)=C$ for all $x \in[S, T)$. Then it is immediate that

$$
\begin{aligned}
\int_{-\infty}^{\infty} f(y) \sum_{x \in[S, T)} 1_{\left\{C-x=y, J\left(\tau_{x}^{L}\right)=j\right\}} \mathrm{d} y & =\int_{-\infty}^{\infty} f(y) 1_{\left\{(C-y) \in[S, T), J\left(\tau_{C-y}^{L}\right)=j\right\}} \mathrm{d} y \\
& =\int_{S}^{T} f(C-x) 1_{\left\{J\left(\tau_{x}^{L}\right)=j\right\}} \mathrm{d} x .
\end{aligned}
$$

Summing over all such intervals yields the statement of the lemma.

Proof of Theorem 4.1. Apply Lemma 4.2 with $f(y)=\mathrm{e}^{\alpha y}$ to Corollary 4.1 to obtain

$$
\begin{aligned}
\int_{0}^{\infty} \mathrm{E}\left[\mathrm{e}^{\alpha X\left(\tau_{x}^{L}\right)} ; J\left(\tau_{x}^{L}\right)\right] \mathrm{d} x= & \Pi^{-} \int_{0}^{\infty} \mathrm{e}^{y\left(\Lambda^{-}-\alpha \mathbb{I}^{-}\right)} \mathrm{d} y \mathrm{e}^{x_{0} \Lambda^{-}} K^{-} \\
& +\Pi^{+} \int_{0}^{\infty} \mathrm{e}^{y\left(\Lambda^{+}+\alpha \mathbb{I}^{+}\right)} \mathrm{d} y \mathrm{e}^{\left(B-x_{0}\right) \Lambda^{+}} \Pi_{+}^{-} \mathrm{e}^{B \Lambda^{-}} K^{-} .
\end{aligned}
$$

Consider the MMBM $(-X(t), J(t))$ started in $B-x_{0}$ to find that

$$
\begin{aligned}
\int_{0}^{\infty} \mathrm{E}\left[\mathrm{e}^{\alpha X\left(\tau_{x}^{U}\right)} ; J\left(\tau_{x}^{U}\right)\right] \mathrm{d} x= & \Pi^{+} \int_{0}^{\infty} \mathrm{e}^{y\left(\Lambda^{+}+\alpha \mathbb{I}^{+}\right)} \mathrm{d} y \mathrm{e}^{\left(B-x_{0}\right) \Lambda^{+}} K^{+} \\
& +\Pi^{-} \int_{0}^{\infty} \mathrm{e}^{y\left(\Lambda^{-}-\alpha \mathbb{I}^{-}\right)} \mathrm{d} y \mathrm{e}^{x_{0} \Lambda^{-}} \Pi_{-}^{+} \mathrm{e}^{B \Lambda^{+}} K^{+} .
\end{aligned}
$$

The integrals on the right-hand sides converge if $\rho^{+}+\alpha<0$ and $\rho^{-}-\alpha<0$, that is, $\alpha \in\left(\rho^{-},-\rho^{+}\right)$. Hence, the left-hand sides converge for $\operatorname{such} \alpha$. Use (4.1) to see that $k^{L}(\alpha)<0$ and $k^{U}(\alpha)<0$, and (4.3) holds.

\subsection{Stationary overflow and unused capacity}

Recall that $J\left(\tau_{x}^{L}\right)$ and $J\left(\tau_{x}^{U}\right)$ are irreducible recurrent Markov chains. Denote the corresponding stationary distributions through $\pi^{L}$ and $\pi^{U}$. Define $\lim _{t \rightarrow \infty} L(t) / t=\kappa^{L}$ and, similarly, $\lim _{t \rightarrow \infty} U(t) / t=\kappa^{U}$, which do not depend on the initial distribution of $(X(0), J(0))$. Noting that $\left\{\tau_{x}^{L}<t\right\}=\{x<L(t)\}$, we write

$$
\kappa^{L} \pi_{i}^{L}=\lim _{t \rightarrow \infty} \frac{1}{t} \int_{0}^{L(t)} 1_{\left\{J\left(\tau_{x}^{L}\right)=i\right\}} \mathrm{d} x=\lim _{t \rightarrow \infty} \frac{1}{t} \int_{0}^{\infty} 1_{\left\{J\left(\tau_{x}^{L}\right)=i, \tau_{x}^{L}<t\right\}} \mathrm{d} x \quad \text { almost surely, }
$$

i.e. we can interpret the vectors $\kappa^{L} \pi^{L}$ and $\kappa^{U} \boldsymbol{\pi}^{U}$ as the mean unused capacity and the mean overflow in a unit of time in stationarity.

These quantities were identified in [7] using martingale calculations and the theory of generalized Jordan chains.

Proposition 4.1. We have

$$
\left(\kappa^{L} \boldsymbol{\pi}^{L}, \kappa^{U} \boldsymbol{\pi}^{U}\right)\left(\begin{array}{cc}
\mathbb{I}^{-} & -\Pi_{-}^{+} \mathrm{e}^{B \Lambda^{+}} \\
-\Pi_{+}^{-} \mathrm{e}^{B \Lambda^{-}} & \mathbb{I}^{+}
\end{array}\right)= \begin{cases}\kappa\left(\mathbf{0}, \boldsymbol{\pi}^{+}\right) & \text {if } \kappa>0 \\
\mathbf{0} & \text { if } \kappa=0 \\
-\kappa\left(\boldsymbol{\pi}^{-}, \mathbf{0}\right) & \text { if } \kappa<0\end{cases}
$$

where $q=0$ and $\pi^{ \pm}$is the stationary distribution associated with $\Lambda^{ \pm}$whenever $\pm \kappa>0$. 
This result is Corollary 5.1 of [7]. Note that (4.5) uniquely identifies $\kappa^{L} \boldsymbol{\pi}^{L}$ and $\kappa^{U} \boldsymbol{\pi}^{U}$ if $\kappa \neq 0$. If, however, $\kappa=0$ then an additional equation is required; see [7] for details. It should be mentioned that stationary overflow and unused capacity for an arbitrary MAP are considered in [5]. The results of this paper however depend on the stationary distribution, which has no explicit solution unless the MAP can be reduced to an MMBM. Moreover, a restrictive assumption about the number of roots of a certain equation is made, which can fail even in the case of an MMBM.

In the following we retrieve the above result by a simple argument using Theorem 4.1. Observe that

$$
-M^{L}(0)=\int_{0}^{\infty} \mathrm{P}\left(\tau_{x}^{L}<e_{q}, J\left(\tau_{x}^{L}\right)\right) \mathrm{d} x
$$

and, hence, by (4.4) we obtain $-\lim _{q \downarrow 0} q\left[M^{L}(0)\right]_{i j}=\kappa^{L} \pi_{j}^{L}$ for any $i \in E$ and $j \in E^{-}$. A similar identity holds if we consider $\tau_{x}^{ \pm}$. Firstly, note that

$$
-\Pi^{+}\left(\Lambda^{+}\right)^{-1}=\int_{0}^{\infty} \mathrm{P}\left(\tau_{x}^{+}<e_{q}, J\left(\tau_{x}^{+}\right)\right) \mathrm{d} x \quad \text { and } \quad\left\{\tau_{x}^{+}<e_{q}\right\}=\left\{x<\bar{X}\left(e_{q}\right)\right\},
$$

where $\bar{X}(t)=\sup \{X(s): 0 \leq s \leq t\}$. Secondly, it is well known that $\lim _{t \rightarrow \infty} \bar{X}(t) / t=$ $\kappa 1_{\{\kappa>0\}}$. Finally, consider (4.2) with $q>0$ and $\alpha=0$, multiply both sides by $q$, and let $q \downarrow 0$ to see that every row of this equation reduces to (4.5).

\subsection{Special cases}

An important special case arises when $E^{-}=E^{+}=E$, that is, there are no states when the process evolves deterministically (a Brownian component is always present). In this special case $\Pi^{-}=\Pi^{+}=\mathbb{I}$ and, hence, (4.3) reduces to

$$
M^{L}(\alpha)=\left(\left(\Lambda^{-}-\alpha \mathbb{I}\right)^{-1} \mathrm{e}^{x_{0} \Lambda^{-}}+\left(\Lambda^{+}+\alpha \mathbb{I}\right)^{-1} \mathrm{e}^{\left(B-x_{0}\right) \Lambda^{+}} \mathrm{e}^{B \Lambda^{-}}\right)\left(\mathbb{I}-\mathrm{e}^{B \Lambda^{+}} \mathrm{e}^{B \Lambda^{-}}\right)^{-1} .
$$

Letting $x_{0}=0$ and taking the inverse we obtain

$$
F^{L}(\alpha)=\left(\mathrm{e}^{-B \Lambda^{-}}-\mathrm{e}^{B \Lambda^{+}}\right)\left(\left(\Lambda^{-}-\alpha \mathbb{I}\right)^{-1} \mathrm{e}^{-B \Lambda^{-}}+\left(\Lambda^{+}+\alpha \mathbb{I}\right)^{-1} \mathrm{e}^{B \Lambda^{+}}\right)^{-1} .
$$

Moreover, observing that $X\left(\tau_{0}^{L}\right)=U\left(\tau_{0}^{L}\right)$ we find from (4.3) that

$$
\begin{aligned}
\mathrm{E}_{x_{0}=B} & {\left[\mathrm{e}^{\alpha U\left(\tau_{0}^{L}\right)-q \tau_{0}^{L}} ; J\left(\tau_{0}^{L}\right)\right] } \\
& =\left(\left(\Lambda^{-}-\alpha \mathbb{I}\right)^{-1}+\left(\Lambda^{+}+\alpha \mathbb{I}\right)^{-1}\right)\left(\left(\Lambda^{-}-\alpha \mathbb{I}\right)^{-1} \mathrm{e}^{-B \Lambda^{-}}+\left(\Lambda^{+}+\alpha \mathbb{I}\right)^{-1} \mathrm{e}^{B \Lambda^{+}}\right)^{-1} \\
& =\left(\Lambda^{-}-\alpha \mathbb{I}\right)^{-1}\left(\Lambda^{+}+\Lambda^{-}\right)\left(\mathrm{e}^{-B \Lambda^{-}}\left(\Lambda^{+}+\alpha \mathbb{I}\right)+\left(\Lambda^{-}-\alpha \mathbb{I}\right) \mathrm{e}^{B \Lambda^{+}}\right)^{-1}\left(\Lambda^{-}-\alpha \mathbb{I}\right),
\end{aligned}
$$

where in the last step we used the fact that $\left(\Lambda^{+}+\alpha \mathbb{I}\right)^{-1}$ and $\mathrm{e}^{B \Lambda^{+}}$commute. A number of other useful transforms can be found using similar algebraic manipulations.

Next, we restrict ourselves to the case of a single state, that is, we consider a Brownian motion $\left(\sigma^{2}, \mu\right)$. Without real loss of generality, it is assumed that $\sigma^{2}=1$. According to (2.2), $\lambda=\Lambda^{ \pm}$is a solution of $1 / 2 \lambda^{2} \mp \mu \lambda-q=0$. Moreover, $\Lambda^{ \pm}$is negative unless $q=0$ and $\pm \mu \geq 0$, in which case it is 0 . Thus, $\Lambda^{+}=\mu-\gamma$ and $\Lambda^{-}=-\mu-\gamma$, where $\gamma=\sqrt{\mu^{2}+2 q}$. Now the right-hand side of (4.7) reduces to $-2 \gamma /\left[\mathrm{e}^{B(\mu+\gamma)}(\mu+\alpha-\gamma)-\mathrm{e}^{B(\mu-\gamma)}(\mu+\alpha+\gamma)\right]$ and so

$$
\mathrm{E}_{B}\left[\mathrm{e}^{\alpha U\left(\tau_{0}^{L}\right)-q \tau_{0}^{L}}\right]=\frac{\mathrm{e}^{-B \mu}}{\cosh (B \gamma)-(\mu+\alpha) \sinh (B \gamma) / \gamma},
$$


where $\alpha \in(-\mu-\gamma,-\mu+\gamma)$ according to Theorem 4.1. Fix $q>0$ for a moment, so that $\gamma>0$. Multiply both sides of the equation by the denominator on the right-hand side and observe that the Laplace transform is analytic in $\operatorname{Re}(\alpha)<-\mu+\gamma$. Thus, the latter equality holds in this domain. This shows that (4.8) holds for all $q>0$ and $\alpha \leq 0$. By symmetry we obtain

$$
\mathrm{E}_{0}\left[\mathrm{e}^{-\alpha L\left(\tau_{0}^{U}\right)-q \tau_{0}^{U}}\right]=\frac{\mathrm{e}^{B \mu}}{\cosh (B \gamma)+(\mu+\alpha) \sinh (B \gamma) / \gamma}, \quad q>0, \alpha \geq 0,
$$

which is Equation (7) of [17]. Taking limits on both sides we show that this equation also holds for $q=0$, unless $\mu=0$, in which case $\mathrm{Ee}^{-\alpha L\left(\tau_{0}^{U}\right)}=1 /(1+\alpha B)$ by the l'Hôpital's rule. Note that Williams [17] used a very different approach, namely stochastic integration and she relied on a sophisticated guess of the right form of a certain function. Our approach, however, is direct and is based on simple probabilistic arguments.

Let us conclude by making some additional comments about the Brownian motion with two reflecting barriers. Firstly, the strong Markov property of $X(t)$ implies that the nondecreasing piecewise constant process $U\left(\tau_{x}^{L}\right)$ has memoryless jumps and interarrival times, implying that it is a Poisson process with exponential jumps. Let us confirm this and find the corresponding rates. Note that (4.6) can be rewritten as

$$
F^{L}(\alpha, q)=\frac{2\left(\alpha^{2} / 2+\mu \alpha-q\right)}{\gamma \operatorname{coth}(B \gamma)-(\mu+\alpha)} .
$$

Hence, if $\mu \neq 0$ then

$$
\log \mathrm{Ee}^{\alpha U\left(\tau_{1}^{L}\right)}=F^{L}(\alpha)+\alpha=\frac{-\alpha \mu(\operatorname{coth}(\mu B)+1)}{\alpha-\mu(\operatorname{coth}(\mu B)-1)},
$$

which implies that $U\left(\tau_{x}^{L}\right)$ jumps with rate $\mu(\operatorname{coth}(\mu B)+1)=2 \mu /\left(1-\mathrm{e}^{-2 \mu B}\right)$, and the jumps are exponential of rate $\mu(\operatorname{coth}(\mu B)-1)=2 \mu /\left(\mathrm{e}^{2 \mu B}-1\right)$. If $\mu=0$ then $\log \operatorname{Ee}^{\alpha U\left(\tau_{1}^{L}\right)}=$ $\alpha /(1-\alpha B)$, that is, both rates become $1 / B$.

\section{Acknowledgements}

I would like to thank Onno Boxma and Michel Mandjes for their comments and suggestions. I am also grateful to Yoni Nazarathy, through whom I became aware of the relevant work by Williams [17].

\section{References}

[1] Ahn, S., Badescu, A. L. And Ramaswami, V. (2007). Time dependent analysis of finite buffer fluid flows and risk models with a dividend barrier. Queueing Systems 55, 207-222.

[2] Anick, D., Mitra, D. And Sondhi, M. M. (1982). Stochastic theory of a data-handling system with multiple sources. Bell System Tech. J. 61, 1871-1894.

[3] Asmussen, S. (1995). Stationary distributions for fluid flow models with or without Brownian noise. Commun. Statist. Stoch. Models 11, 21-49.

[4] Asmussen, S. (2003). Applied Probability and Queues (Appl. Math. 51), 2nd edn. Springer, New York.

[5] Asmussen, S. And Pihlsgånd, M. (2007). Loss rates for Lévy processes with two reflecting barriers. Math. Operat. Res. 32, 308-321.

[6] Breuer, L. (2008). First passage times for Markov additive processes with positive jumps of phase type. J. Appl. Prob. 45, 779-799.

[7] D’Auria, B., Ivanovs, J., Kella, O. and Mandjes, M. (2010). First passage process of a Markov additive process, with applications to reflection problems. Preprint. Available at http://arxiv.org/abs/1006.2965v1. 
[8] Ivanovs, J. And Mandjes, M. (2010). First passage of time-reversible spectrally negative Markov additive processes. Operat. Res. Lett. 38, 77-81.

[9] Karandikar, R. L. AND Kulkarni, V. G. (1995). Second-order fluid flow models: reflected Brownian motion in a random environment. Operat. Res. 43, 77-88.

[10] Kosten, L. (1974/75). Stochastic theory of a multi-entry buffer. I. Delft Progress Rep. 1, 10-18.

[11] Kruk, L., Lehoczky, J., Ramanan, K. and Shreve, S. (2007). An explicit formula for the Skorokhod map on [0, a]. Ann. Prob. 35, 1740-1768.

[12] Kulkarni, V. G. (1997). Fluid models for single buffer systems. In Frontiers in Queueing, ed. J. H. Dshalalow, CRC, Boca Raton, FL, pp. 321-338.

[13] Lindley, D. (1959). Discussion on Mr. Winsten's paper. J. R. Statist. Soc. B 21, 22-23.

[14] Pistorius, M. (2006). On maxima and ladder processes for a dense class of Lévy processes. J. Appl. Prob. 43, 208-220.

[15] Prabhu, N. U. and Zhu, Y. (1989). Markov-modulated queueing systems. Queueing Systems 5, $215-245$.

[16] Rogers, L. C. G. (1994). Fluid models in queueing theory and Wiener-Hopf factorization of Markov chains. Ann. Appl. Prob. 4, 390-413.

[17] Williams, R. J. (1992). Asymptotic variance parameters for the boundary local times of reflected Brownian motion on a compact interval. J. Appl. Prob. 29, 996-1002. 